\title{
Optimal Power Flow Using Tabu Search Algorithm
}

\author{
M. A. ABIDO \\ Electrical Engineering Department \\ King Fahd University of Petroleum and Minerals \\ Dhahran 31261, Saudi Arabia
}

This paper presents an efficient and reliable tabu search (TS)-based approach to solve the optimal power flow (OPF) problem. The proposed approach employs $T S$ algorithm for optimal settings of the control variables of the OPF problem. Incorporation of TS as a derivative-free optimization technique in solving OPF problem significantly reduces the computational burden. One of the main advantages of TS algorithm is its robustness to its own parameter settings as well as the initial solution. In addition, TS is characterized by its ability to avoid entrapment in local optimal solution and prevent cycling by using flexible memory of search history. The proposed approach has been examined on the standard IEEE 30-bus test system with different objectives and generator cost curves. The results are promising and show the effectiveness and robustness of the proposed approach.

Keywords optimal power flow, tabu search algorithm, combinatorial optimization

\section{Introduction}

In the past two decades, the problem of optimal power flow (OPF) has received much attention. It is of current interest of many utilities, and it has been marked as one of the most operational needs. The OPF problem solution aims to optimize a selected objective function, such as fuel cost via optimal adjustment of the power system control variables, while at the same time satisfying various equality and inequality constraints. The equality constraints are the power flow equations, and the inequality constraints are the limits on control variables and the operating limits of power system-dependent variables. The problem control variables include the generator real powers, the generator bus voltages, the transformer tap settings, and the reactive power of switchable VAR sources, while the problem-dependent variables include the load bus voltages, the generator reactive powers, and the line flows. Generally, the OPF problem is a large-scale, highly constrained, nonlinear, nonconvex optimization problem.

Mathematically speaking, OPF problem is a large-scale combinatorial optimization problem, known as the NP-complete problem [1]. It has been shown that no optimal solution to a large-scale NP-complete problem can be obtained using

Manuscript received in final form on 29 May 2001.

The author gratefully acknowledges the support of King Fahd University of Petroleum \& Minerals.

Address correspondence to M. A. Abido. 
affordable solution time. Besides, the OPF problem even has a nondifferentiable objective function, which induces further difficulties when seeking an optimal solution to the problem.

A wide variety of optimization techniques have been applied to solving the OPF problems [2-20], such as nonlinear programming (NLP) [2-6], quadratic programming (QP) [7, 8], linear programming (LP) [9-11], Newton-based techniques [12-13], sequential unconstrained minimization technique (SUMT) [14], and interior point methods $[15,16]$. Generally, NLP-based procedures have many drawbacks, such as insecure convergence properties and algorithmic complexity. QP-based techniques have some disadvantages associated with the piecewise quadratic cost approximation. Newton-based techniques have a drawback of the convergence characteristics that are sensitive to the initial conditions, and they may even fail to converge due to the inappropriate initial conditions. SUMT methods are known to exhibit numerical difficulties when the penalty factors become extremely large. Although LP methods are fast and reliable, they have some disadvantages associated with the piecewise linear cost approximation. Interior point methods have been reported as computationally efficient; however, if the step size is not chosen properly, the sublinear problem may have a solution that is infeasible in the original nonlinear domain [15]. In addition, interior point methods, in general, suffer from bad initial, termination, and optimality criteria and, in most cases, are unable to solve nonlinear and quadratic objective functions [16]. For more discussions on these techniques, we direct the reader to consult the comprehensive survey presented in [17].

Generally, most of these approaches apply sensitivity analysis and gradientbased optimization algorithms by linearizing the objective function and the system constraints around an operating point. Unfortunately, the problem of the OPF is a highly nonlinear and a multimodal optimization problem (i.e., there exist more than one local optimum). Hence, local optimization techniques, which are well elaborated, are not suitable for such a problem. Moreover, there is no local criterion to decide whether a local solution is also the global solution. Therefore, conventional optimization methods that make use of derivatives and gradients, in general, are not able to locate or identify the global optimum. On the other hand, many mathematical assumptions, such as convex, analytic, and differential objective functions, have to be given to simplify the problem; however, the OPF problem is an optimization problem with, in general, nonconvex, nonsmooth, and nondifferentiable objective functions. These properties become more evident and dominant if the effects of the valve-point loading of thermal generators and the nonlinear behavior of electronic-based devices, such as FACTS, are taken into consideration. Hence, it becomes essential to develop optimization techniques that are efficient to overcome these drawbacks and handle such difficulties.

Recently, heuristic algorithms, such as genetic algorithms (GA) [18] and evolutionary programming [19], have been proposed for solving the OPF problem. The results reported were promising and encouraging for further research in this direction. In the last few years, TS algorithm appeared as another promising heuristic algorithm for handling the combinatorial optimization problems [20-22]. TS has been applied to various power system optimization problems with impressive success [23-25].

In this paper, TS algorithm is proposed to solve the OPF problem. Unlike other heuristic techniques, the advantage of using TS algorithm is the fact that it uses a flexible memory of search history to prevent cycling and to avoid entrapment 
in local optima. Unlike the traditional optimization techniques, TS can easily deal with nonconvex, nonsmooth, and nondifferentiable objective functions. It has been theoretically proved that tabu search algorithm can yield global optimal solution with probability one [22].

\section{Problem Formulation}

The optimal power flow problem is to optimize the steady state performance of a power system in terms of an objective function while satisfying several equality and inequality constraints. Mathematically, the OPF problem can be formulated as follows:

$$
\operatorname{Min} J(\mathbf{x}, \mathbf{u})
$$

subject to

$$
\begin{aligned}
& g(\mathbf{x}, \mathbf{u})=0 \\
& h(\mathbf{x}, \mathbf{u}) \leq 0
\end{aligned}
$$

where

$\mathbf{x}$ is the vector of dependent variables consisting of slack bus power $P_{G_{1}}$, load bus voltages $V_{L}$, generator reactive power outputs $Q_{G}$, and transmission line loadings $S_{l}$. Hence, $\mathbf{x}$ can be expressed as $\mathbf{x}^{T}=\left[P_{G_{1}}, V_{L}, Q_{G}, S_{l}\right]$ (i.e., $\mathbf{x}^{T}=$ $\left[P_{G_{1}}, V_{L_{1}} \ldots V_{L_{N L}}, Q_{G_{1}} \ldots Q_{G_{N} G}, S_{l 1} \ldots S_{l_{n l}}\right]$ ), where $N L, N G$, and $n l$ are number of load buses, number of generators, and number of transmission lines, respectively.

$\mathbf{u}$ is the vector of independent variables consisting of generator voltages $V_{G}$, generator real power outputs $P_{G}$ except at the slack bus $P_{G_{1}}$, and transformer tap settings $T$. Hence, $\mathbf{u}$ can be expressed as $\mathbf{u}^{T}=\left[V_{G}, P_{G}, T\right]$ (i.e., $\mathbf{u}^{T}=$ $\left.\left[V_{G_{1}} \ldots V_{G_{N G}}, P_{G_{2}} \ldots P_{G_{N} G}, T_{1} \ldots T_{N T}\right]\right)$, where $N T$ is the number of the regulating transformers.

$J$ is the objective function to be minimized. Generally, for OPF problem, the objective function $J$ is that of total fuel cost; i.e.,

$$
J=\sum_{i=1}^{N G} f_{i} \quad(\$ / \mathrm{h}),
$$

where $f_{i}$ is the fuel cost of the $i$ th generator.

$g$ is the equality constraints and represent typical load flow equations.

$h$ is the system operating constraints that include:

(a) Generation constraints: Generator voltages, real power outputs, and reactive power outputs are restricted by the lower and upper limits as follows:

$$
\begin{array}{ll}
V_{G_{i}}^{\min } \leq V_{G_{i}} \leq V_{G_{i}}^{\max }, & i \in N G, \\
P_{G_{i}}^{\min } \leq P_{G_{i}} \leq P_{G_{i}}^{\max }, & i \in N G, \\
Q_{G_{i}}^{\min } \leq Q_{G_{i}} \leq Q_{G_{i}}^{\max }, & i \in N G .
\end{array}
$$


(b) Transformer constraints: Transformer tap settings are restricted by the lower and upper limits; i.e.,

$$
T_{i}^{\min } \leq T_{i} \leq T_{i}^{\max }, \quad i \in N T .
$$

(c) Security constraints: These include the constraints of voltages at load buses and transmission line loadings as follows:

$$
\begin{aligned}
V_{L_{i}}^{\min } & \leq V_{L_{i}} \leq V_{L_{i}}^{\max }, \quad i \in N L, \\
S_{l_{i}} & \leq S_{l_{i}}^{\max }, \quad i \in n l .
\end{aligned}
$$

It is worth mentioning that the control variables are self-constrained. The hard inequalities of $P_{G_{1}}, V_{L}, Q_{G}$, and $S_{l}$ can be incorporated in the objective function as quadratic penalty terms. Therefore, the objective function can be augmented as follows:

$$
\begin{aligned}
J= & \sum_{i=1}^{N G} f_{i}+\lambda_{P}\left(P_{G_{1}}-P_{G 1}^{\lim }\right)^{2}+\lambda_{V} \sum_{i=1}^{N L}\left(V_{L_{i}}-V_{L_{i}}^{\lim }\right)^{2} \\
& +\lambda_{Q} \sum_{i=1}^{N G}\left(Q_{G_{i}}-Q_{G_{i}}^{\lim }\right)^{2}+\lambda_{S} \sum_{i=1}^{n l}\left(S_{l_{i}}-S_{l_{i}}^{\max }\right)^{2}
\end{aligned}
$$

where $\lambda_{P}, \lambda_{V}, \lambda_{Q}$, and $\lambda_{S}$ are the penalty factors, and $x^{\lim }$ is the limit value of the dependent variable $x$ given as

$$
x^{\lim }=\left\{\begin{array}{ll}
x^{\max } ; & x>x^{\max } \\
x^{\min } ; & x<x^{\min }
\end{array} .\right.
$$

\section{Tabu Search Algorithm}

\subsection{Overview}

Tabu search is a higher-level heuristic algorithm for solving combinatorial optimization problems. It is an iterative improvement procedure that starts from any initial solution and attempts to determine a better solution. TS was proposed in its present form a few years ago [20-22]. It has now become an established optimization approach that is rapidly spreading to many new fields. Together with other heuristic search algorithms, such as GA, TS has been singled out as "extremely promising" for the future treatment of practical applications [20]. Generally, the advantages of TS over other traditional optimization techniques can be summarized as follows:

- TS is characterized by its ability to avoid entrapment in local optimal solution and prevent cycling by using flexible memory of search history.

- TS uses payoff (performance index or objective function) information to guide the search in the problem space. Therefore, it can easily deal with nonsmooth, noncontinuous, and nondifferentiable objective functions that are the real-life optimization problems. Additionally, this property relieves TS of assumptions and approximations, which often are required by traditional optimization methods for many practical optimization problems. 
- TS uses probabilistic transition rules to make decisions, not deterministic rules. Hence, TS is a kind of stochastic optimization algorithm that can search a complicated and uncertain area to find the global optimum. This makes TS more flexible and robust than conventional methods.

Typically, the TS algorithm starts with no knowledge of the correct solution, depending entirely on responses from interacting environment to arrive at optimal solution.

\subsection{TS A lgorithm}

The basic elements of TS are briefly stated and defined as follows:

- Current solution, $x_{\text {current }}$ : It is a set of the optimized parameter values at any iteration. It plays a central role in generating the neighbor trial solutions.

- Moves: They characterize the process of generating trial solutions that are related to $x_{\text {current }}$.

- Set of candidate moves, $N\left(x_{\text {current }}\right)$ : It is the set of all possible moves or trial solutions, $x_{\text {trial }} \mathrm{s}$, in the neighborhood of $x_{\text {current }}$. In case of continuous variable optimization problems, this set is too large or even infinite set. Therefore, one could operate with a subset, $S\left(x_{\text {current }}\right)$, with a limited number of trial solutions, $n t$, of this set (i.e., $S \subset N$ and $x_{\text {trial }} \in S\left(x_{\text {current }}\right)$ ).

- Tabu restrictions: These are certain conditions imposed on moves that make some of them forbidden. These forbidden moves are listed to a certain size and known as tabu. This list is called the tabu list. The reason behind classifying a certain move as forbidden is basically to prevent cycling and avoid returning to the local optimum just visited. The tabu list size plays a great role in the search of high-quality solutions. The way to identify a good tabu list size is to simply watch for the occurrence of cycling when the size is too small and the deterioration in solution quality when the size is too large, caused by forbidding too many moves.

- Aspiration Criterion (Level): It is a rule that overrides tabu restrictions (i.e., if a certain move is forbidden by tabu restriction, the aspiration criterion, when satisfied, can make this move allowable). Different forms of aspiration criteria are used in the literature [20-22]. The one considered here is to override the tabu status of a move if this move yields a solution which has better objective function, $J$, than the one obtained earlier with the same move. The importance of using aspiration criterion is to add some flexibility in the TS by directing it toward the attractive moves.

- Stopping Criteria: These are the conditions under which the search process will terminate. In this study, the search will terminate if one of the following criteria is satisfied: (a) the number of iterations since the last change of the best solution is greater than a prespecified number; (b) the number of iterations reaches the maximum allowable number.

The general algorithm of TS can be described in steps as follows:

Step 1: Set the iteration counter $k=0$ and randomly generate an initial solution $x_{\text {initial }}$. Set this solution as the current solution as well as the best solution, $x_{\text {best }}$ (i.e., $x_{\text {initial }}=x_{\text {current }}=x_{\text {best }}$ ). 
Step 2: Randomly generate a set of trial solutions, $x_{\text {trial }}$, in the neighborhood of the current solution (i.e., create $\left.S\left(x_{\text {current }}\right)\right)$. Sort the elements of $S$ based on their objective function values in ascending order, as the problem is a minimization one. Let us define $x_{\text {trial }}^{i}$ as the $i$ th trial solution in the sorted set, $1 \leq i \leq n t$. Here, $x_{\text {trial }}^{1}$ represents the best trial solution in $S$ in terms of objective function value associated with it.

Step 3: Set $i=1$. If $J\left(x_{\text {trial }}^{i}\right)>J\left(x_{\text {best }}\right)$, go to Step 4, else set $x_{\text {best }}=x_{\text {trial }}^{i}$ and go to Step 4.

Step 4: Check the tabu status of $x_{\text {trial }}^{i}$. If it is not in the tabu list, then put it in the tabu list, set $x_{\text {current }}=x_{\text {trial }}^{i}$, and go to Step 7. If it is in tabu list, go to Step 5.

Step 5: Check the aspiration criterion of $x_{\text {trial }}^{i}$. If satisfied, then override the tabu restrictions, update the aspiration level, set $x_{\text {current }}=x_{\text {trial }}^{i}$, and go to Step 7. If not, set $i=i+1$ and go to Step 6 .

Step 6: If $i>n t$, go to Step 7, else go back to Step 4 .

Step 7: Check the stopping criteria. If one of them is satisfied, then stop, else set $k=k+1$ and go back to Step 2 .

The computational flow of TS algorithm is shown in Figure 1.

\subsection{Application of TS to OPF Problem}

The above-described TS algorithm is excited by generating randomly initial values of the optimized control variables (i.e., initial solution). Then, the objective function is evaluated. The search for the optimal set of the control variables will continue until one of the stopping criteria is satisfied. In our implementation, the search will terminate if (a) the number of iterations since the last change of the best solution is greater than 50 iterations, or (b) the number of iterations reaches the maximum allowable number of 500 .

\section{Numerical Results}

The proposed TS-based OPF algorithm has been applied to the standard IEEE 30-bus test system shown in Figure 2. The system line and bus data are given in the Appendix. The lower limit of voltage magnitude for all busses is 0.95 p.u. The upper limit of voltage magnitude is 1.05 p.u. for slack bus and all load busses, while the upper limit of all other generator busses is 1.1 p.u. The lower and upper limits of all transformer taps are 0.9 and 1.1 p.u., respectively.

In order to demonstrate the effectiveness and robustness of the technique, several cases with different objectives and generator cost curves have been considered as follows.

\subsection{Case (a): Quadratic Cost Curve}

In this case, the generator cost curves are represented by quadratic functions as

$$
f_{i}=a_{i}+b_{i} P_{G_{i}}+c_{i} P_{G_{i}}^{2} \quad(\$ / \mathrm{h}),
$$

where $a_{i}, b_{i}$, and $c_{i}$ are the cost coefficients of the $i$ th generator. The values of these coefficients are given in Table 1. 


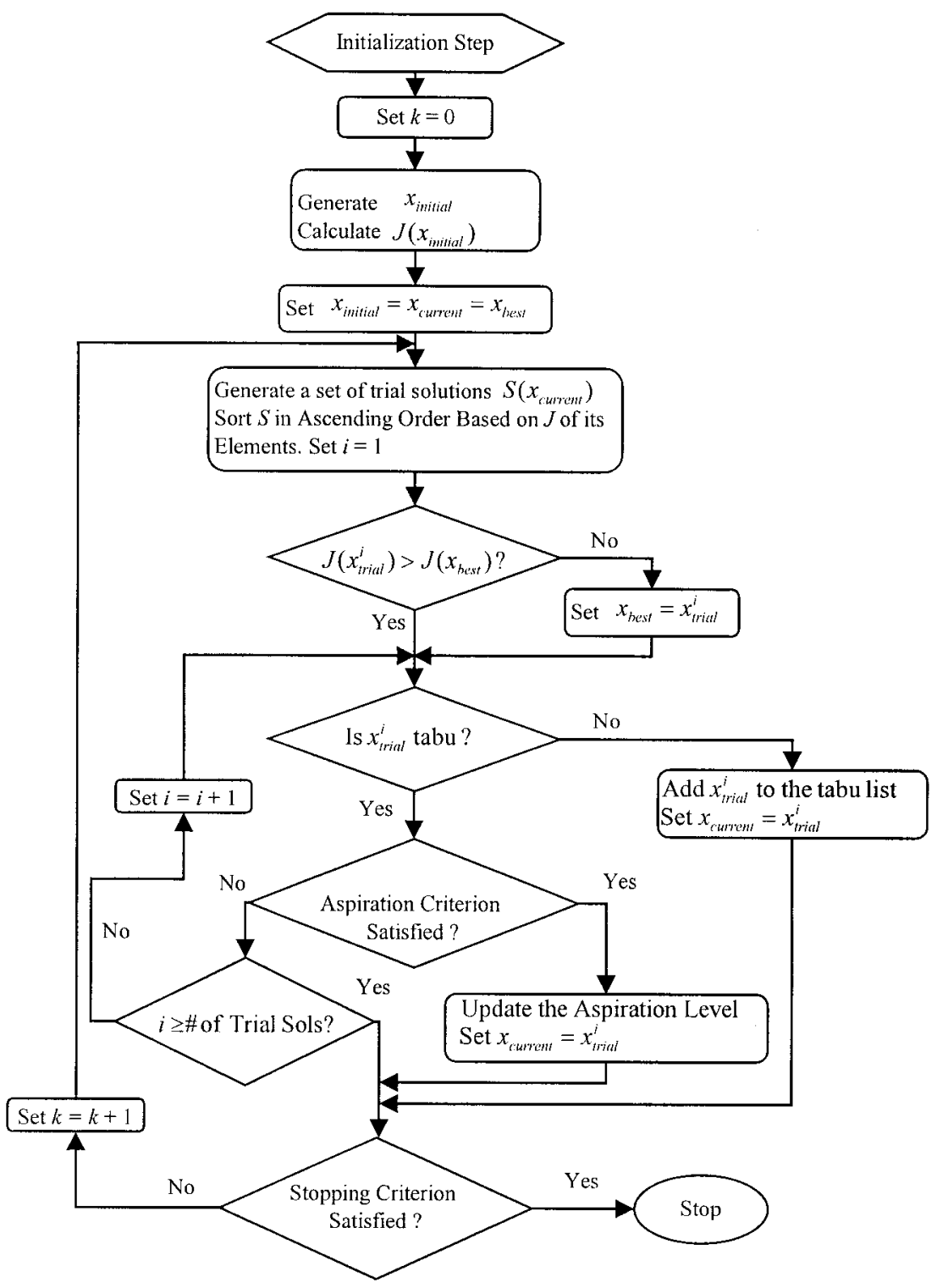

Figure 1. The computational flow of TS algorithm.

The technique was tested for several initial solutions. Different initial solutions are generated by changing the seed of the random number generator. The variations of the objective function with different initialization are shown in Figure 3. It is clear that the technique converges to the optimal solution regardless of the initial one. The optimal values of the control variables are given in Table 3. The total cost obtained by the proposed technique is $\$ 802.29$, while the best obtained after 100 runs by evolutionary programming was $\$ 802.62$ [19]. In addition, the nonlinear programming solution is $\$ 802.40$ [3]. This confirms the superiority of the proposed technique over the evolutionary programming and nonlinear programming techniques. 


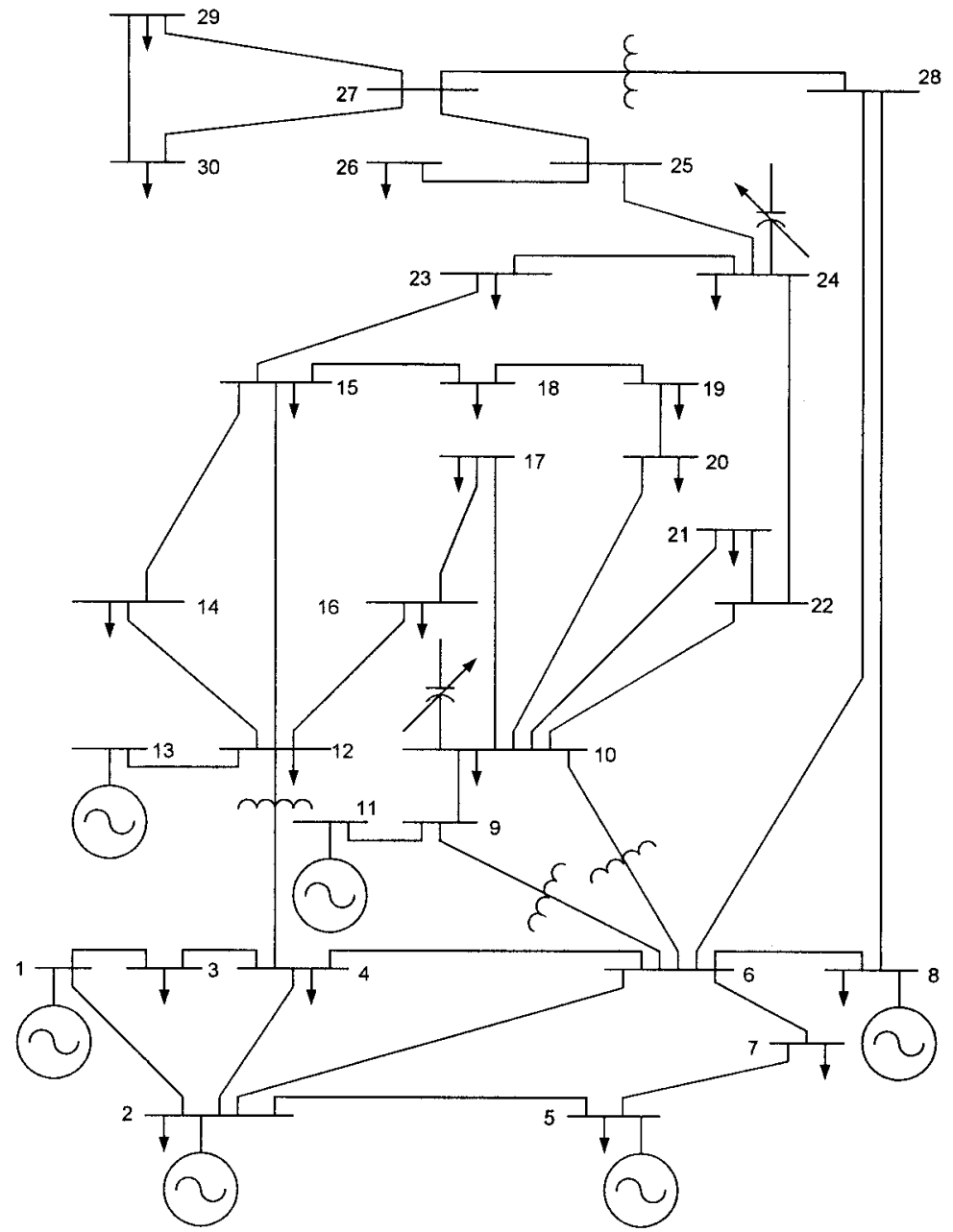

Figure 2. Single-line diagram of IEEE 30-bus test system.

Table 1

Generator data and cost coefficients for case (a)

\begin{tabular}{|c|c|c|c|c|c|c|c|}
\hline \multirow[b]{2}{*}{ Bus } & \multirow{2}{*}{$\begin{array}{l}P_{G}^{\min } \\
(\mathrm{pu})\end{array}$} & \multirow{2}{*}{$\begin{array}{c}P_{G}^{\max } \\
(\mathrm{pu})\end{array}$} & \multirow{2}{*}{$\begin{array}{l}Q_{G}^{\min } \\
(\mathrm{pu})\end{array}$} & \multirow{2}{*}{$\begin{array}{l}S_{G}^{\max } \\
(\mathrm{pu})\end{array}$} & \multicolumn{3}{|c|}{ Cost coefficients } \\
\hline & & & & & $\mathrm{a}$ & $\mathrm{b}$ & c \\
\hline 1 & 0.50 & 2.00 & -0.20 & 2.50 & 0.0 & 200 & 37.5 \\
\hline 2 & 0.20 & 0.80 & -0.20 & 1.00 & 0.0 & 175 & 175 \\
\hline 5 & 0.15 & 0.50 & -0.15 & 0.80 & 0.0 & 100 & 625 \\
\hline 8 & 0.10 & 0.35 & -0.15 & 0.60 & 0.0 & 325 & 83.4 \\
\hline 11 & 0.10 & 0.30 & -0.10 & 0.50 & 0.0 & 300 & 250 \\
\hline 13 & 0.12 & 0.40 & -0.15 & 0.60 & 0.0 & 300 & 250 \\
\hline
\end{tabular}




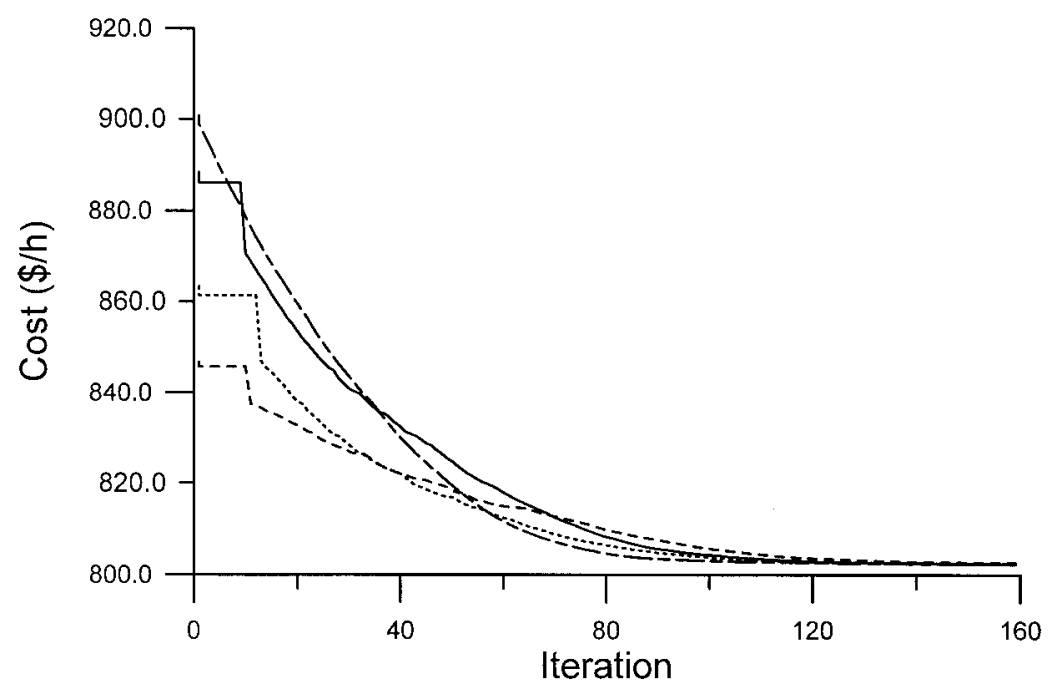

Figure 3. Objective function variation for different initialization of case (a).

\subsection{Case (b): Quadratic Cost Curve with Sine Components}

Approximating the cost curves by smooth quadratic functions results in some inaccuracy due to neglecting the ripples produced by the valve point loading. In this case, a sine component is superimposed to the cost curves of the generators at buses 1 and 2 to reflect the valve-point loading effects [26] as

$$
f_{i}=a_{i}+b_{i} P_{G_{i}}+c_{i} P_{G_{i}}^{2}+d_{i} \mid \sin \left(e_{i}\left(P_{G_{i}}^{\min }-\left(P_{G_{i}}\right)\right) \mid \quad(\$ / \mathrm{h}),\right.
$$

where $a_{i}, b_{i}, c_{i}, d_{i}$, and $e_{i}$ are the cost coefficients of the $i$ th generator. The values of these coefficients are given in Table 2 [19]. The cost curves of the other generators are the same as in case (a). The technique was tested with different initializations. The objective function variations are shown in Figure 4. It is evident that the technique converges to the optimal solution regardless of the initial one. The optimal values of control variables are given in Table 2 . The total cost obtained by the proposed technique is $\$ 919.715$, while the minimum cost obtained by EP after 100 runs was $\$ 919.89$. It is clear that the proposed technique outperforms the evolutionary programming.

Table 2

Generator cost coefficients for case (b)

\begin{tabular}{cccrcccc}
\hline & & & \multicolumn{5}{c}{ Cost coefficients } \\
\cline { 4 - 8 } Bus & $\begin{array}{l}P_{G}^{\min } \\
(\mathrm{pu})\end{array}$ & \begin{tabular}{l}
$P_{G}^{\max }(\mathrm{pu})$ \\
\cline { 4 - 8 }
\end{tabular} & $\mathrm{a}$ & $\mathrm{b}$ & $\mathrm{c}$ & $\mathrm{d}$ & $\mathrm{e}$ \\
\hline 1 & 0.50 & 2.00 & 150 & 200 & 16 & 50 & 6.3 \\
2 & 0.20 & 0.80 & 25 & 250 & 100 & 40 & 9.8 \\
\hline
\end{tabular}




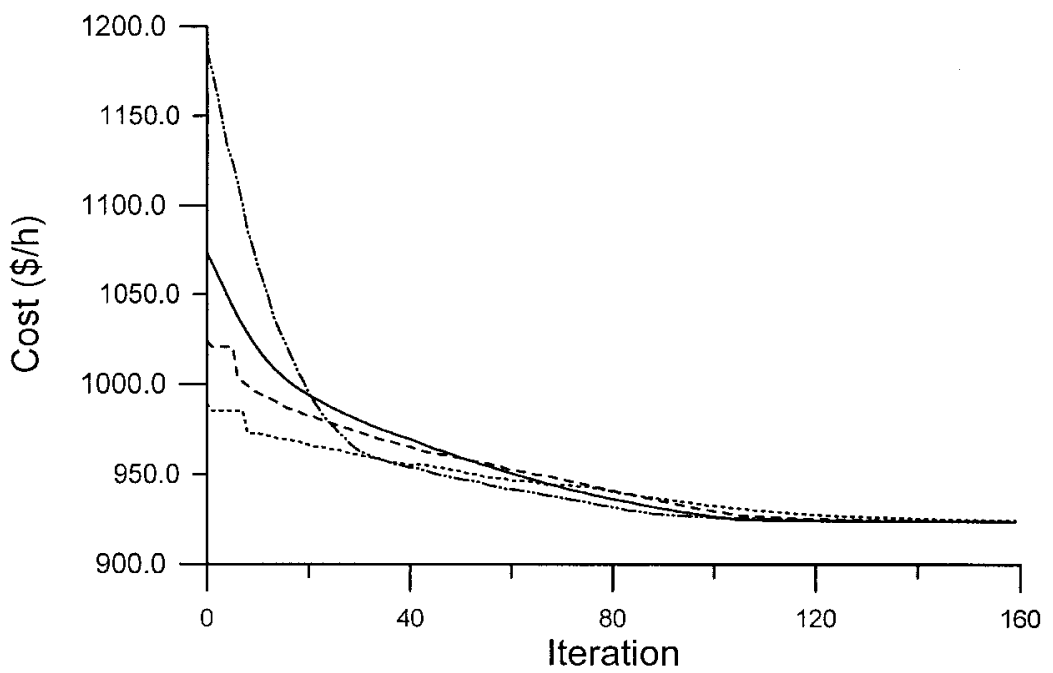

Figure 4. Objective function variation for different initialization of case (b).

\subsection{Case (c): Voltage Profile Optimization}

Bus voltage is one of the most important security and service quality indices. Considering only cost-based objectives in OPF problem may result in a feasible solution that has unattractive voltage profile. In this case, a twofold objective function is considered in order to minimize the fuel cost and improve voltage profile by minimizing the load bus voltage deviations from 1.0 per unit. The objective function can be expressed as

$$
\begin{aligned}
J= & \sum_{i=1}^{N G} f_{i}+w \sum_{i \in N L}\left|v_{i}-1.0\right|+\lambda_{P}\left(P_{G_{1}}-P_{G_{1}}^{\lim }\right)^{2}+\lambda_{V} \sum_{i=1}^{N L}\left(V_{L_{i}}-V_{L_{i}}^{\lim }\right)^{2} \\
& +\lambda_{Q} \sum_{i=1}^{N G}\left(Q_{G_{i}}-Q_{G_{i}}^{\lim }\right)^{2}+\lambda_{S} \sum_{i=1}^{n l}\left(S_{l_{i}}-S_{l_{i}}^{\mathrm{max}}\right)^{2}
\end{aligned}
$$

where $w$ is a weighting factor. In this case, the generator cost curves are the same as in case (a). The optimal settings of the control variables are given in Table 3. The system voltage profile compared to that of case (a) is shown in Figure 5. It is evident that the voltage profile is greatly improved compared to that of case (a). Specifically, the total sum of voltage deviations is reduced from 0.7615 in case (a) to 0.1405 in case (c). This gives a reduction ratio of $81.55 \%$. The total generation cost in this case, however, is slightly increased by $0.58 \%$ from that of case (a).

\subsection{Case (d): Solution Quality Improvement}

The results shown in Figures 2 and 3 emphasize that the proposed approach finally leads to the optimal control variable settings regardless of the initial one. Based on this conclusion, the proposed approach can be used to improve the solution quality of other methods. To demonstrate this point, case (a) is reconsidered with two different initial solutions. First, the initial solution of the proposed approach is set 
Table 3

Optimal values of control variables

\begin{tabular}{ccccc}
\hline & Initial & Case (a) & Case (b) & Case (c) \\
\hline$P_{1}$ & 0.9880 & 1.7604 & 2.0000 & 1.7288 \\
$P_{2}$ & 0.8000 & 0.4876 & 0.3965 & 0.4894 \\
$P_{5}$ & 0.5000 & 0.2156 & 0.2042 & 0.2032 \\
$P_{8}$ & 0.2000 & 0.2205 & 0.1247 & 0.2448 \\
$P_{11}$ & 0.2000 & 0.1244 & 0.1000 & 0.1496 \\
$P_{13}$ & 0.2000 & 0.1200 & 0.1200 & 0.1222 \\
$V_{1}$ & 1.0500 & 1.0500 & 1.0500 & 1.0157 \\
$V_{2}$ & 1.0450 & 1.0389 & 1.0342 & 1.0034 \\
$V_{5}$ & 1.0100 & 1.0110 & 1.0118 & 1.0195 \\
$V_{8}$ & 1.0100 & 1.0198 & 1.0185 & 1.0095 \\
$V_{11}$ & 1.0500 & 1.0941 & 1.0868 & 1.0201 \\
$V_{13}$ & 1.0500 & 1.0898 & 1.0942 & 1.0430 \\
$T_{11}$ & 0.9780 & 1.0407 & 0.9993 & 1.0424 \\
$T_{12}$ & 0.9690 & 0.9218 & 1.0017 & 0.9016 \\
$T_{15}$ & 0.9320 & 1.0098 & 1.0184 & 0.9946 \\
$T_{36}$ & 0.9680 & 0.9402 & 0.9586 & 0.9448 \\
$J$ & $\mathbf{9 0 0 . 7 6}$ & $\mathbf{8 0 2 . 2 9}$ & $\mathbf{9 1 9 . 7 2}$ & $\mathbf{8 0 6 . 9 8}$ \\
\hline
\end{tabular}

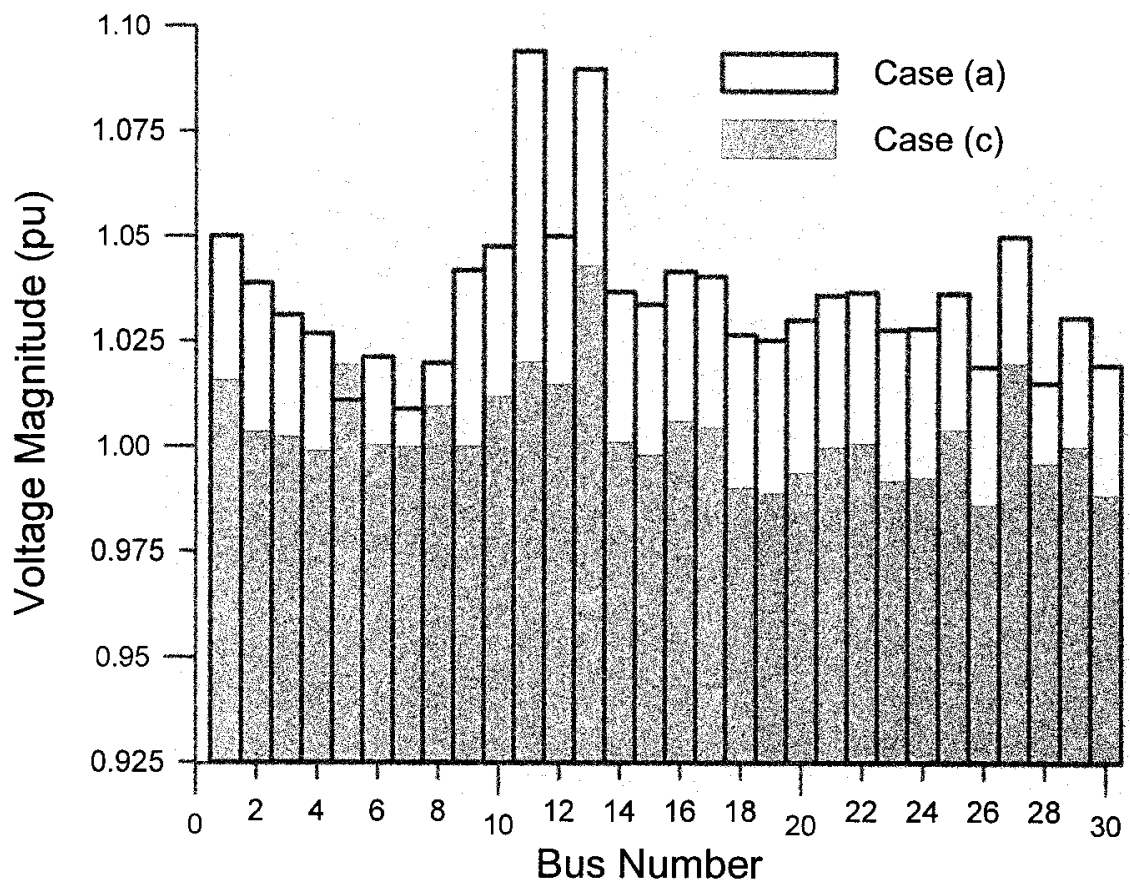

Figure 5. System voltage profile for case (c). 


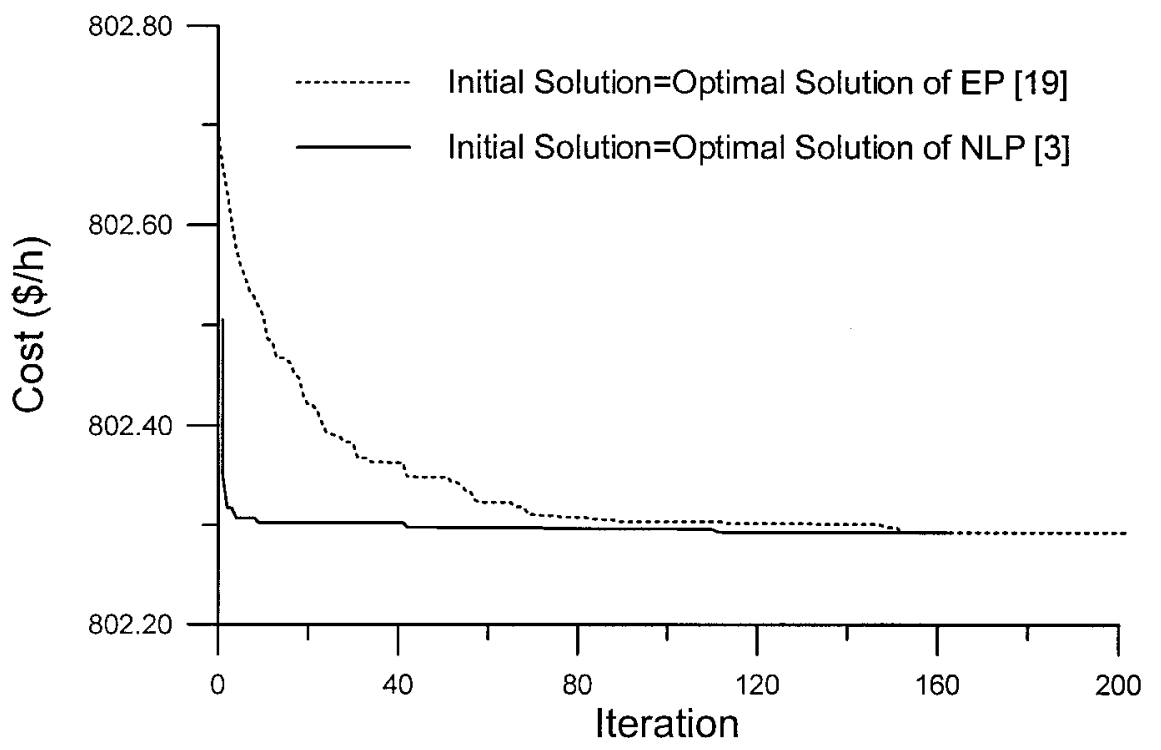

Figure 6. Improving the quality of a given optimal solution.

as the optimal solution of the evolutionary programming approach given in [19]. Second, the initial solution of the proposed approach is set as the optimal solution of the nonlinear programming approach given in [3]. The quality improvement of the given optimal solutions of other methods is shown in Figure 6. The results show the capability of the proposed approach to improve the quality of the optimal solution of other traditional methods. It is worth mentioning that the proposed approach converges to the optimal solution of case (a) given in Table 3 with these different initializations. It can be concluded that the proposed approach can be hybridized with other methods in order to improve the optimal solution quality.

\section{Conclusion}

In this paper, a TS-based approach to optimal power flow problem has been presented. The proposed approach has been tested and examined with different objectives and different classes of generator cost functions to demonstrate its effectiveness and robustness. The results using the proposed approach were compared to that reported using evolutionary programming and nonlinear programming techniques. It is clear that the proposed approach outperforms the classical and evolutionary algorithms. The major advantage of the proposed approach is its robustness to the initial solution. Therefore, the proposed approach can be used to improve the solution quality obtained by other classical techniques.

\section{References}

[1] C. Papadimitriou, 1982, Combinatorial Optimization: Algorithms and Complexity, Prentice Hall.

[2] H. Dommel and W. Tinny, 1968, "Optimal Power Flow Solution," IEEE Trans. on Power Apparatus and Systems, Vol. PAS-87, No. 10, pp. 1866-1876. 
[3] O. Alsac and B. Stott, 1974, "Optimal Load Flow with Steady State Security," IEEE Trans. on Power Apparatus and Systems, Vol. PAS-93, pp. 745-751.

[4] R. Shoults and D. Sun, 1982, "Optimal Power Flow Based on P-Q Decomposition," IEEE Trans. on Power Apparatus and Systems, Vol. PAS-101, No. 2, pp. 397-405.

[5] H. H. Happ, 1977, "Optimal Power Dispatch: A Comprehensive Survey," IEEE Trans. on Power Apparatus and Systems, Vol. PAS-96, pp. 841-854.

[6] K. R. C. Mamandur and R. D. Chenoweth, 1981, "Optimal Control of Reactive Power Flow for Improvements in Voltage Profiles and for Real Power Loss Minimization," IEEE Trans. on Power Apparatus and Systems, Vol. PAS-100, No. 7, pp. 31853193.

[7] R. C. Burchett, H. H. Happ, and K. A. Wirgau, 1982, "Large Scale Optimal Power Flow," IEEE Trans. on Power Apparatus and Systems, Vol. PAS-101, pp. 3722-3732.

[8] K. Aoki, A. Nishikori, and R. T. Yokoyama, 1987, "Constrained Load Flow Using Recursive Quadratic Programming," IEEE Trans. on Power Systems, Vol. 2, No. 1, pp. 8-16.

[9] A. A. Abou El-Ela and M. A. Abido, 1992, "Optimal Operation Strategy for Reactive Power Control," Modelling, Simulation \& Control, Part A, AMSE Press, Vol. 41, No. 3, pp. 19-40.

[10] W. O. Stadlin and D. L. Fletcher, 1982, "Voltage Versus Reactive Current Model for Dispatch and Control," IEEE Trans. on Power Apparatus and Systems, Vol. PAS-101, No. 10 , pp. 3751-3758.

[11] R. Mota-Palomino and V. H. Quintana, 1986, "Sparse Reactive Power Scheduling by a Penalty-Function Linear Programming Technique," IEEE Trans. on Power Systems, Vol. 1, No. 3, pp. 31-39.

[12] D. I. Sun, B. Ashley, B. Brewer, A. Hughes, and W. F. Tinney, 1984, "Optimal Power Flow by Newton Approach," IEEE Trans. on Power Apparatus and Systems, Vol. PAS-103, No. 10, pp. 2864-2875.

[13] A. Santos and G. R. da Costa, 1995, "Optimal Power Flow Solution by Newton's Method Applied to an Augmented Lagrangian Function," IEE Proc.-Gener. Transm. Distrib., Vol. 142, No. 1, pp. 33-36.

[14] M. Rahli and P. Pirotte, 1999, "Optimal Load Flow Using Sequential Unconstrained Minimization Technique (SUMT) Method under Power Transmission Losses Minimization," Electric Power Systems Research, Vol. 52, pp. 61-64.

[15] X. Yan and V. H. Quintana, 1999, "Improving an Interior Point based OPF by Dynamic Adjustments of Step Sizes and Tolerances," IEEE Trans. on Power Systems, Vol. 14, No. 2, pp. 709-717.

[16] J. A. Momoh and J. Z. Zhu, 1999, “Improved Interior Point Method for OPF Problems," IEEE Trans. on Power Systems, Vol. 14, No. 3, pp. 1114-1120.

[17] J. A. Momoh, M. E. El-Hawary, and R. Adapa, 1999, "A Review of Selected Optimal Power Flow Literature to 1993 Parts I \& II,” IEEE Trans. on Power Systems, Vol. 14, No. 1, pp. 96-111.

[18] L. L. Lai and J. T. Ma, 1997, "Improved Genetic Algorithms for Optimal Power Flow Under Both Normal and Contingent Operation States," Int. J. Electrical Power \& Energy Systems, Vol. 19, No. 5, pp. 287-292.

[19] J. Yuryevich and K. P. Wong, 1999, "Evolutionary Programming Based Optimal Power Flow Algorithm," IEEE Trans. on Power Systems, Vol. 14, No. 4, pp. 12451250.

[20] J. A. Bland and G. Dawson, 1991, “Tabu Search and Design Optimization," ComputerAided Design, Vol. 23, No. 3, pp. 195-201.

[21] F. Glover, 1989, “Tabu Search-Part I,” ORSA Journal on Computing, Vol. 1, No. 3, pp. 190-206.

[22] F. Glover, 1990, “Tabu Search-Part II," ORSA Journal on Computing, Vol. 2, No. 1, pp. 4-32. 
[23] M. A. Abido, 1999, "A Novel Approach to Conventional Power System Stabilizer Design Using Tabu Search,” International Journal of Electrical Power \& Energy Systems, Vol. 21, No. 6, pp. 443-454.

[24] F. S. Wen and C. S. Chang, 1997, "Tabu Search Approach to Alarm Processing in Power Systems," IEE Proc. Genet. Transm. Distrib., Vol. 144, No. 1, pp. 31-38.

[25] X. Bai and S. M. Shahidehpour, 1996, "Hydro-Thermal Scheduling by Tabu Search and Decomposition Method," IEEE Trans. PWRS, Vol. 11, No. 2, pp. 968-974.

[26] D. C. Walters and G. B. Sheble, 1993, "Genetic Algorithm Solution of Economic Dispatch with Valve Point Loading," IEEE Trans. PWRS, Vol. 8, No. 3, pp. 13251332.

\section{Appendix}

Table A.1

IEEE 30-bus test system line data

\begin{tabular}{|c|c|c|c|c|c|c|}
\hline Line & From & To & $R$ (p.u.) & $X$ (p.u.) & $B$ (p.u.) & Rating (p.u.) \\
\hline 1 & 1 & 2 & 0.0192 & 0.0575 & 0.0264 & 1.30 \\
\hline 2 & 1 & 3 & 0.0452 & 0.1852 & 0.0204 & 1.30 \\
\hline 3 & 2 & 4 & 0.0570 & 0.1737 & 0.0184 & 0.65 \\
\hline 4 & 3 & 4 & 0.0132 & 0.0379 & 0.0042 & 1.30 \\
\hline 5 & 2 & 5 & 0.0472 & 0.1983 & 0.0209 & 1.30 \\
\hline 6 & 2 & 6 & 0.0581 & 0.1763 & 0.0187 & 0.65 \\
\hline 7 & 4 & 6 & 0.0119 & 0.0414 & 0.0045 & 0.90 \\
\hline 8 & 5 & 7 & 0.0460 & 0.1160 & 0.0102 & 0.70 \\
\hline 9 & 6 & 7 & 0.0267 & 0.0820 & 0.0085 & 1.30 \\
\hline 10 & 6 & 8 & 0.0120 & 0.0420 & 0.0045 & 0.32 \\
\hline 11 & 6 & 9 & 0.0000 & 0.2080 & 0.0000 & 0.65 \\
\hline 12 & 6 & 10 & 0.0000 & 0.5560 & 0.0000 & 0.32 \\
\hline 13 & 9 & 11 & 0.0000 & 0.2080 & 0.0000 & 0.65 \\
\hline 14 & 9 & 10 & 0.0000 & 0.1100 & 0.0000 & 0.65 \\
\hline 15 & 4 & 12 & 0.0000 & 0.2560 & 0.0000 & 0.65 \\
\hline 16 & 12 & 13 & 0.0000 & 0.1400 & 0.0000 & 0.65 \\
\hline 17 & 12 & 14 & 0.1231 & 0.2559 & 0.0000 & 0.32 \\
\hline 18 & 12 & 15 & 0.0662 & 0.1304 & 0.0000 & 0.32 \\
\hline 19 & 12 & 16 & 0.0945 & 0.1987 & 0.0000 & 0.32 \\
\hline 20 & 14 & 15 & 0.2210 & 0.1997 & 0.0000 & 0.16 \\
\hline 21 & 16 & 17 & 0.0824 & 0.1923 & 0.0000 & 0.16 \\
\hline 22 & 15 & 18 & 0.1070 & 0.2185 & 0.0000 & 0.16 \\
\hline 23 & 18 & 19 & 0.0639 & 0.1292 & 0.0000 & 0.16 \\
\hline 24 & 19 & 20 & 0.0340 & 0.0680 & 0.0000 & 0.32 \\
\hline 25 & 10 & 20 & 0.0936 & 0.2090 & 0.0000 & 0.32 \\
\hline 26 & 10 & 17 & 0.0324 & 0.0845 & 0.0000 & 0.32 \\
\hline 27 & 10 & 21 & 0.0348 & 0.0749 & 0.0000 & 0.32 \\
\hline 28 & 10 & 22 & 0.0727 & 0.1499 & 0.0000 & 0.32 \\
\hline 29 & 21 & 22 & 0.0116 & 0.0236 & 0.0000 & 0.32 \\
\hline
\end{tabular}


Table A.1

(Continued)

\begin{tabular}{ccccccc}
\hline Line & From & To & $R$ (p.u.) & $X$ (p.u.) & $B$ (p.u.) & Rating (p.u.) \\
\hline 30 & 15 & 23 & 0.1000 & 0.2020 & 0.0000 & 0.16 \\
31 & 22 & 24 & 0.1150 & 0.1790 & 0.0000 & 0.16 \\
32 & 23 & 24 & 0.1320 & 0.2700 & 0.0000 & 0.16 \\
33 & 24 & 25 & 0.1885 & 0.3292 & 0.0000 & 0.16 \\
34 & 25 & 26 & 0.2544 & 0.3800 & 0.0000 & 0.16 \\
35 & 25 & 27 & 0.1093 & 0.2087 & 0.0000 & 0.16 \\
36 & 28 & 27 & 0.0000 & 0.3960 & 0.0000 & 0.65 \\
37 & 27 & 29 & 0.2198 & 0.4153 & 0.0000 & 0.16 \\
38 & 27 & 30 & 0.3202 & 0.6027 & 0.0000 & 0.16 \\
39 & 29 & 30 & 0.2399 & 0.4533 & 0.0000 & 0.16 \\
40 & 8 & 28 & 0.0636 & 0.2000 & 0.0214 & 0.32 \\
41 & 6 & 28 & 0.0169 & 0.0599 & 0.0065 & 0.32 \\
\hline
\end{tabular}

Table A.2

IEEE 30-bus test system bus data

\begin{tabular}{rrrrrr}
\hline Bus & $P($ MW $)$ & Q (MVAR $)$ & Bus & $P($ MW $)$ & Q (MVAR) \\
\hline 1 & 0.00 & 0.00 & 16 & 3.50 & 1.80 \\
2 & 21.70 & 12.70 & 17 & 9.00 & 5.80 \\
3 & 2.40 & 1.20 & 18 & 3.20 & 0.90 \\
4 & 7.60 & 1.60 & 19 & 9.50 & 3.40 \\
5 & 94.20 & 19.00 & 20 & 2.20 & 0.70 \\
6 & 0.00 & 0.00 & 21 & 17.50 & 11.20 \\
7 & 22.80 & 10.90 & 22 & 0.00 & 0.00 \\
8 & 30.00 & 30.00 & 23 & 3.20 & 1.60 \\
9 & 0.00 & 0.00 & 24 & 8.70 & 6.70 \\
10 & 5.80 & 2.00 & 25 & 0.00 & 0.00 \\
11 & 0.00 & 0.00 & 26 & 3.50 & 2.30 \\
12 & 11.20 & 7.50 & 27 & 0.00 & 0.00 \\
13 & 0.00 & 0.00 & 28 & 0.00 & 0.00 \\
14 & 6.20 & 1.60 & 29 & 2.40 & 0.90 \\
15 & 8.20 & 2.50 & 30 & 10.60 & 1.90 \\
\hline
\end{tabular}

